\title{
Towards the Total Synthesis of Natural Peloruside A: Stereoselective Synthesis of the Backbone of the Core
}

\author{
Bo Liu and Wei-Shan Zhou* \\ Shanghai Institute of Organic Chemistry, Chinese Academy of Sciences, 354 Fenglin Road, \\ Shanghai 200032, P. R. China
}

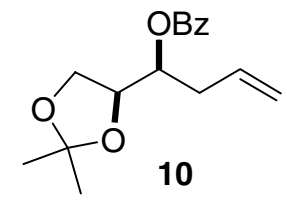

${ }^{1} \mathrm{HNMR}\left(300 \mathrm{MHz}, \mathrm{CDCl}_{3}\right)$ ठ 8.05-8.08 (m, 2H), 7.53-7.59 (m, 1H), 7.41-7.47 (m, 2H), $5.76-$ $5.89(\mathrm{~m}, 1 \mathrm{H}), 5.22-5.28(\mathrm{~m}, 1 \mathrm{H}), 5.05-5.18(\mathrm{~m}, 2 \mathrm{H}), 4.30-4.36(\mathrm{~m}, 1 \mathrm{H}), 4.06(\mathrm{dd}, \mathrm{J}=8.4,6.9 \mathrm{~Hz}, \mathrm{~A}$ of $\mathrm{ABX}, 1 \mathrm{H}), 3.81(\mathrm{dd}, \mathrm{J}=8.4,6.6 \mathrm{~Hz}, \mathrm{~B}$ of $\mathrm{ABX}, 1 \mathrm{H}), 2.49-2.58(\mathrm{~m}, 2 \mathrm{H}), 1.46(\mathrm{~s}, 3 \mathrm{H}), 1.37(\mathrm{~s}, 3 \mathrm{H})$; ${ }^{13} \mathrm{CNMR}\left(75 \mathrm{MHz}, \mathrm{CDCl}_{3}\right) \delta 166.1,133.0$ (2C), 130.1, 129.7, 128.3, 118.4, 109.6, 76.1, 72.9, 65.6, 35.4, 26.3, 25.4; [a $]_{\mathrm{D}}{ }^{25}=-16.8\left(\mathrm{c} 0.93, \mathrm{CHCl}_{3}\right)$; IR (film, $\left.\mathrm{cm}^{-1}\right)$ 1719, 1645; EI-MS: 261 $\left(\left[\mathrm{M}-\mathrm{CH}_{3}\right]^{+}\right)$.

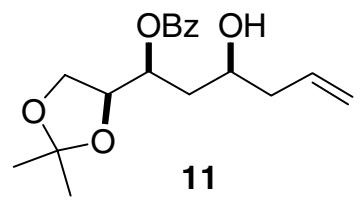

${ }^{1} \mathrm{HNMR}\left(300 \mathrm{MHz}, \mathrm{CDCl}_{3}\right)$ ठ 8.05-8.09 (m, 2H), 7.55-7.61 (m, 1H), 7.42-7.47 (m, 2H), 5.74 $5.88(\mathrm{~m}, 1 \mathrm{H}), 5.33-5.38(\mathrm{~m}, 1 \mathrm{H}), 5.10-5.15(\mathrm{~m}, 2 \mathrm{H}), 4.39-4.45(\mathrm{~m}, 1 \mathrm{H}), 4.09$ (dd, J=8.7, 6.6 Hz, A of ABX, 1H), 3.85-3.96 (m, 1H), 3.83 (dd, J=8.4, 6.6 Hz, B of ABX, 1H), 2.15-2.40 (m, 2H), 1.84 2.05 (m, 2H), 1.48 (s, 3H), 1.38 (s, 3H); ${ }^{13} \mathrm{CNMR}\left(75 \mathrm{MHz}, \mathrm{CDCl}_{3}\right)$ ठ 166.2, 134.2, 133.1, 129.9, $129.7,128.4,118.3,109.7,76.2,71.3,67.2,65.6,42.0,37.8,26.2,25.2 ;[\alpha]_{\mathrm{D}}{ }^{25}=-9.1(\mathrm{c} 1.01$, $\mathrm{CHCl}_{3}$ ); IR (film, $\left.\mathrm{cm}^{-1}\right)$ 1720, 1642; ESI-MS: $343.1\left([\mathrm{M}+\mathrm{Na}]^{+}\right)$.

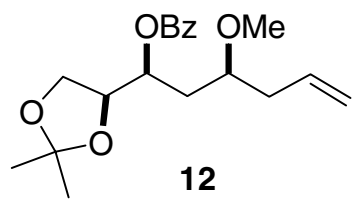

${ }^{1} \mathrm{HNMR}\left(300 \mathrm{MHz}, \mathrm{CDCl}_{3}\right) \delta$ 8.02-8.06 (m, 2H), 7.54-7.58 (m, 1H), 7.41-7.47 (m, 2H), 5.78-5.89 
(m, 1H), 5.32-5.38 (m, 1H), 5.08-5.17 (m, 2H), 4.21-4.28 (m, 1H), $4.00(\mathrm{dd}, \mathrm{J}=8.1,6.6 \mathrm{~Hz}$, A of ABX, 1H), 3.72 (dd, J=7.8, 6.6 Hz, B of ABX, 1H), $3.41(\mathrm{~s}, 3 \mathrm{H}), 3.33-3.44(\mathrm{~m}, 1 \mathrm{H}), 2.47-2.55(\mathrm{~m}$, $2 \mathrm{H}), 1.86-1.91$ (m, 2H), 1.43 (s, 3H), 1.33 (s, 3H); ${ }^{13} \mathrm{CNMR}\left(75 \mathrm{MHz}, \mathrm{CDCl}_{3}\right.$ ) $\delta 165.8,133.1$, $132.7,130.2,129.3,128.2,118.2,109.2,78.5,76.6,71.2,65.4,57.9,38.6,33.5,26.2,24.9 ;[\alpha]_{D}{ }^{25}=$ +6.2 (c 1.21, $\left.\mathrm{CHCl}_{3}\right)$; IR (film, $\left.\mathrm{cm}^{-1}\right)$ 1718, 1643; ESI-MS: $335.1\left([\mathrm{M}+\mathrm{H}]^{+}\right), 357.1\left([\mathrm{M}+\mathrm{Na}]^{+}\right)$.

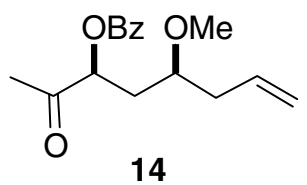

${ }^{1} \mathrm{HNMR}\left(300 \mathrm{MHz}, \mathrm{CDCl}_{3}\right) \delta$ 8.00-8.03 (m, 2H), 7.54-7.56 (m, 1H), $7.44(\mathrm{t}, \mathrm{J}=7.5 \mathrm{~Hz}, 2 \mathrm{H})$, 5.77-5.91 (m, 1H), 5.31-5.36 (m, 1H), 5.09-5.17 (m, 2H), 3.72-3.76 (m, 1H), $3.34(\mathrm{~s}, 3 \mathrm{H})$, 2.47-2.52 (m, 2H), 2.11 (s, 3H), 2.02-2.20 (m, 2H); ${ }^{13} \mathrm{CNMR}\left(75 \mathrm{MHz}, \mathrm{CDCl}_{3}\right) \delta$ 210.5, 165.8, $132.9(2 \mathrm{C}), 130.2,129.5,128.3,118.4,84.2,70.5,57.9,38.5,35.0,25.5 ; ;[\alpha]_{D}{ }^{27}=+3.4(\mathrm{c} 0.50$, $\mathrm{CHCl}_{3}$ ); IR (film, $\left.\mathrm{cm}^{-1}\right)$ 1719, 1643; ESI-MS: $277.1\left([\mathrm{M}+\mathrm{H}]^{+}\right)$.

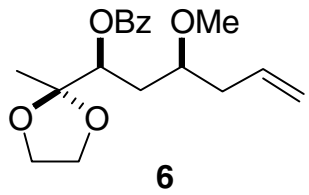

${ }^{1} \mathrm{HNMR}\left(300 \mathrm{MHz}, \mathrm{CDCl}_{3}\right) \delta$ 8.04-8.07 (m, 2H), 7.53-7.57 (m, 1H), 7.41-7.46 (m, 2H), 5.79-5.92 $(\mathrm{m}, 1 \mathrm{H}), 5.34-5.42(\mathrm{~m}, 1 \mathrm{H}), 5.07-5.16(\mathrm{~m}, 2 \mathrm{H}), 3.88-3.99(\mathrm{~m}, 4 \mathrm{H}), 3.42(\mathrm{~s}, 3 \mathrm{H}), 3.23(\mathrm{dd}, \mathrm{J}=7.8,3.9$ $\mathrm{Hz}, 1 \mathrm{H}), 2.40-2.60(\mathrm{~m}, 2 \mathrm{H}), 1.84-1.99(\mathrm{~m}, 2 \mathrm{H}), 1.30(\mathrm{~s}, 3 \mathrm{H}) ;{ }^{13} \mathrm{CNMR}\left(75 \mathrm{MHz}, \mathrm{CDCl}_{3}\right) \delta$ 166.0, $133.4,132.7,130.6,129.5,128.3,118.0,111.1,81.6,72.4,64.9,64.7,59.2,38.7,35.0,19.5 ;[\alpha]_{\mathrm{D}}{ }^{27}$ $=+13.9\left(\mathrm{c} 1.21, \mathrm{CHCl}_{3}\right) ; \mathrm{IR}\left(\right.$ film, $\left.\mathrm{cm}^{-1}\right)$ 1718, 1643; ESI-MS: $321.0\left([\mathrm{M}+\mathrm{H}]^{+}\right), 343.1\left([\mathrm{M}+\mathrm{Na}]^{+}\right)$.

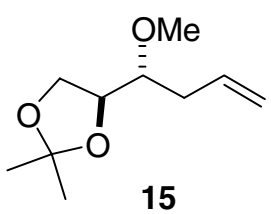

${ }^{1} \mathrm{HNMR}\left(300 \mathrm{MHz}, \mathrm{CDCl}_{3}\right) \delta$ 5.83-5.94 (m, 1H), 5.08-5.17 (m, 2H), 4.00-4.06 (m, 2H), 3.86-3.91 (m, 1H), $3.43(\mathrm{~s}, 3 \mathrm{H}), 3.28-3.33(\mathrm{~m}, 1 \mathrm{H}), 2.27-2.40(\mathrm{~m}, 2 \mathrm{H}), 1.42(\mathrm{~s}, 3 \mathrm{H}), 1.35(\mathrm{~s}, 3 \mathrm{H}) ;{ }^{13} \mathrm{CNMR}$ $\left(75 \mathrm{MHz}, \mathrm{CDCl}_{3}\right) \delta 134.0,117.3,108.9,80.9,76.8,66.3,58.2,34.8,26.5,25.2 ; \mathrm{IR}\left(\mathrm{film}, \mathrm{cm}^{-1}\right.$ ) 
1642; EI-MS: $171\left(\left[\mathrm{M}-\mathrm{CH}_{3}\right]^{+}\right)$.

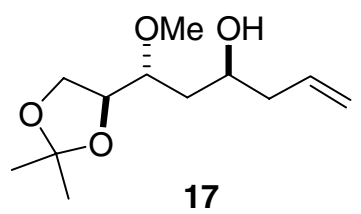

${ }^{1} \mathrm{HNMR}\left(300 \mathrm{MHz}, \mathrm{CDCl}_{3}\right)$ ठ 5.79-5.93 (m, 1H), 5.09-5.18 (m, 2H), 4.06-4.16 (m, 2H), 3.84-3.93 (m, 2H), 3.47-3.50 (m, 1H), 3.47 (s, 3H), 2.26-2.27 (m, 2H), 1.65-1.70 (m, 2H), $1.43(\mathrm{~s}, 3 \mathrm{H}), 1.36$ (s, 3H); ${ }^{13} \mathrm{CNMR}\left(75 \mathrm{MHz}, \mathrm{CDCl}_{3}\right) \delta$ 134.7, 117.8, 109.3, 80.0, 77.1, 67.7, 66.7, 58.7, 42.4, 37.1, 26.4, 25.2; $[\alpha]_{\mathrm{D}}^{25}=+11.2\left(\mathrm{c} 0.49, \mathrm{CHCl}_{3}\right)$; IR (film, $\left.\mathrm{cm}^{-1}\right)$ 1642, 1088, 1063; ESI-MS: 253.1 $\left([\mathrm{M}+\mathrm{Na}]^{+}\right)$.

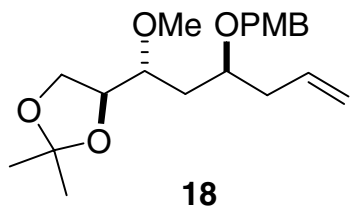

${ }^{1} \mathrm{HNMR}\left(300 \mathrm{MHz}, \mathrm{CDCl}_{3}\right) \delta 7.27$ (d, J=9.0 Hz, 2H), 6.87 (d, J=9.0 Hz, 2H), 5.75-5.91 (m,1H), 5.06-5.14 (m, 2H), $4.59(\mathrm{~d}, \mathrm{~J}=10.8 \mathrm{~Hz}, \mathrm{~A}$ of $\mathrm{AB}, 1 \mathrm{H}), 4.37(\mathrm{~d}, \mathrm{~J}=10.8 \mathrm{~Hz}, \mathrm{~B}$ of $\mathrm{AB}, 1 \mathrm{H}), 4.09-4.15$ (m, 1H), 3.95-4.00 (m, 1H), 3.79-3.84 (m, 1H), $3.79(\mathrm{~s}, 3 \mathrm{H}), 3.65-3.74(\mathrm{~m}, 1 \mathrm{H}), 3.55(\mathrm{dt}, \mathrm{J}=10.5$, $3.3 \mathrm{~Hz}, 1 \mathrm{H}), 3.39(\mathrm{~s}, 3 \mathrm{H}), 2.32-2.41(\mathrm{~m}, 2 \mathrm{H}), 1.43-1.63(\mathrm{~m}, 2 \mathrm{H}), 1.42(\mathrm{~s}, 3 \mathrm{H}), 1.34(\mathrm{~s}, 3 \mathrm{H})$; ${ }^{13} \mathrm{CNMR}\left(75 \mathrm{MHz}, \mathrm{CDCl}_{3}\right) \delta$ 159.1, 134.3, 130.6, 129.4, 117.4, 113.7, 108.9, 77.9, 77.2, 74.3, 70.5, 65.2, 59.3, 55.2, 38.4, 36.3, 26.1, 25.1; IR (film, $\mathrm{cm}^{-1}$ ) 1641, 1614, 1515, 1249; ESI-MS: $373.2\left([\mathrm{M}+\mathrm{Na}]^{+}\right)$.

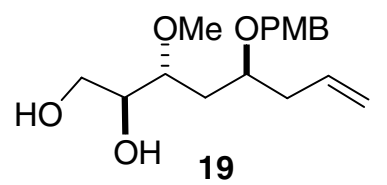

${ }^{1} \mathrm{HNMR}\left(300 \mathrm{MHz}, \mathrm{CDCl}_{3}\right) \delta 7.26(\mathrm{~d}, \mathrm{~J}=9.0 \mathrm{~Hz}, 2 \mathrm{H}), 6.88(\mathrm{~d}, \mathrm{~J}=9.0 \mathrm{~Hz}, 2 \mathrm{H}), 5.75-5.89(\mathrm{~m}, 1 \mathrm{H})$, 5.09-5.15 (m, 2H), $4.61(\mathrm{~d}, \mathrm{~J}=11.1 \mathrm{~Hz}, \mathrm{~A}$ of AB, 1H), $4.36(\mathrm{~d}, \mathrm{~J}=11.1 \mathrm{~Hz}, \mathrm{~B}$ of AB, 1H), $3.80(\mathrm{~s}, 3 \mathrm{H})$, 3.58-3.80 (m, 4H), 3.40-3.44 (m, 1H), 3.33 (s, 3H), 2.33-2.42 (m, 2H), 1.59-1.69 (m, 2H); ${ }^{13} \mathrm{CNMR}$ $\left(75 \mathrm{MHz}, \mathrm{CDCl}_{3}\right) \delta 159.3,134.0,130.1,129.6,117.8,113.8,79.9,75.1,72.6,70.6,63.3,58.2$, 
55.3, 38.2, 35.6; $[\alpha]_{\mathrm{D}}^{23}=+37.0\left(\mathrm{c} 1.26, \mathrm{CHCl}_{3}\right)$; IR (film, $\left.\mathrm{cm}^{-1}\right) 1641,1613,1515,1249$; EI-MS: $310\left(\mathrm{M}^{+}\right)$.

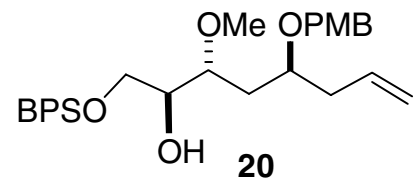

${ }^{1} \mathrm{HNMR}\left(300 \mathrm{MHz}, \mathrm{CDCl}_{3}\right) \delta$ 7.64-7.68 (m, 4H), 7.32-7.45 (m, 6H), 7.24 (d, J=9.0 Hz, 2H), 6.84 $(\mathrm{d}, \mathrm{J}=9.0 \mathrm{~Hz}, 2 \mathrm{H}), 5.74-5.86(\mathrm{~m}, 1 \mathrm{H}), 5.03-5.12(\mathrm{~m}, 2 \mathrm{H}), 4.56(\mathrm{~d}, \mathrm{~J}=11.1 \mathrm{~Hz}, \mathrm{~A}$ of AB, 1H), $4.35(\mathrm{~d}$, $\mathrm{J}=11.1 \mathrm{~Hz}, \mathrm{~B}$ of $\mathrm{AB}, 1 \mathrm{H}), 3.78(\mathrm{~s}, 3 \mathrm{H}), 3.62-3.81(\mathrm{~m}, 4 \mathrm{H}), 3.46-3.51(\mathrm{~m}, 1 \mathrm{H}), 3.26(\mathrm{~s}, 3 \mathrm{H})$, 2.32-2.39 (m, 2H), 1.57-1.61 (m, 2H), 1.06 (s, 9H); ${ }^{13} \mathrm{CNMR}\left(75 \mathrm{MHz}, \mathrm{CDCl}_{3}\right.$ ) ठ 159.0, 135.5, 134.4, 133.1, 130.7, 129.7, 129.4, 127.7, 117.4, 113.7, 78.0, 74.6, 72.8, 70.5, 64.4, 58.1, 55.2, 38.5, 35.4, 26.8, 19.2; IR (film, $\left.\mathrm{cm}^{-1}\right)$ 1641, 1614, 1514, 1249, 1113; ESI-MS: $571.3 \quad\left([\mathrm{M}+\mathrm{Na}]^{+}\right)$.

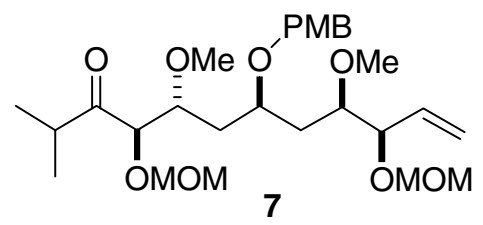

${ }^{1} \mathrm{HNMR}\left(300 \mathrm{MHz}, \mathrm{CDCl}_{3}\right) \delta 7.26(\mathrm{~d}, \mathrm{~J}=8.4 \mathrm{~Hz}, 2 \mathrm{H}), 6.87(\mathrm{~d}, \mathrm{~J}=8.4 \mathrm{~Hz}, 2 \mathrm{H}), 5.71-5.83(\mathrm{~m}, 1 \mathrm{H})$, 5.26-5.31 (m, 2H), $4.70\left(\mathrm{~d}, \mathrm{~J}=6.9 \mathrm{~Hz}, \mathrm{~A}\right.$ of $\mathrm{AB}, \mathrm{A}^{\prime}$ of $\left.\mathrm{A}^{\prime} \mathrm{B}^{\prime}, 2 \mathrm{H}\right), 4.59(\mathrm{~d}, \mathrm{~J}=6.9 \mathrm{~Hz}, \mathrm{~B}$ of $\mathrm{AB}, 1 \mathrm{H})$, $4.58(\mathrm{~d}, \mathrm{~J}=6.9 \mathrm{~Hz}, \mathrm{~B}$ ' of A'B', 1H), 4.52 (d, J=11.4 Hz, A" of A”B", 1H), $4.46($ br. d, J=3.0 Hz, 1H), 4.31 (d, J=11.4 Hz, B" of A"B", 1H), 4.08-4.12 (m, 1H), 3.80 (s, 3H), 3.65-3.77 (m, 2H), 3.44 (s, $3 \mathrm{H}), 3.38(\mathrm{~s}, 3 \mathrm{H}), 3.36(\mathrm{~s}, 3 \mathrm{H}), 3.27-3.34(\mathrm{~m}, 1 \mathrm{H}), 3.23(\mathrm{~s}, 3 \mathrm{H}), 2.82-2.91(\mathrm{~m}, 1 \mathrm{H}), 1.61-1.92(\mathrm{~m}$, 4H), $1.10(\mathrm{~d}, \mathrm{~J}=6.9 \mathrm{~Hz}, 3 \mathrm{H}), 1.05(\mathrm{~d}, \mathrm{~J}=6.9 \mathrm{~Hz}, 3 \mathrm{H}) ;{ }^{13} \mathrm{CNMR}\left(75 \mathrm{MHz}, \mathrm{CDCl}_{3}\right) \delta$ 213.5, 159.0, 134.7, 130.9, 129.5, 118.8, 113.6, 96.3, 94.0, 80.3, 79.9, 78.3 (2C), 72.1, 69.8, 59.0, 57.6, 55.7, 55.6, $55.2,37.7,36.3,35.1,18.6,17.6 ;[\alpha]_{\mathrm{D}}{ }^{27}=+1.1\left(\mathrm{c} 0.53, \mathrm{CHCl}_{3}\right) ; \mathrm{IR}\left(\right.$ film, $\left.\mathrm{cm}^{-1}\right) 1723$ (shoulder), 1614, 1515, 1249; ESI-MS: $535.2\left([\mathrm{M}+\mathrm{Na}]^{+}\right)$.

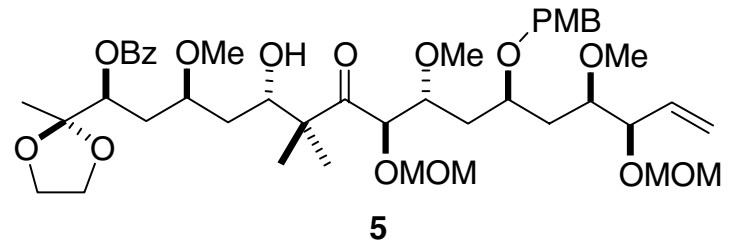


${ }^{1} \mathrm{HNMR}\left(300 \mathrm{MHz}, \mathrm{CDCl}_{3}\right)$ ठ 8.04-8.07 (m, 2H), 7.57-7.61 (m, 1H), 7.42-7.47 (m, 2H), 7.25 (d, $\mathrm{J}=8.7 \mathrm{~Hz}, 2 \mathrm{H}), 6.85(\mathrm{~d}, \mathrm{~J}=8.7 \mathrm{~Hz}, 2 \mathrm{H}), 5.71-5.82(\mathrm{~m}, 1 \mathrm{H}), 5.48-5.58(\mathrm{~m}, 1 \mathrm{H}), 5.25-5.30(\mathrm{~m}, 2 \mathrm{H})$, $4.80(\mathrm{~d}, \mathrm{~J}=3.3 \mathrm{~Hz}, 1 \mathrm{H}), 4.69$ (d, J=6.9 Hz, A of AB, 1H), 4.54-4.60 (m, 3H), 4.49(d, J=11.4 Hz, A' of A'B', 1H), 4.28 (d, J=11.4 Hz, B' of A'B', 1H), 4.05-4.10 (m, 1H), 3.86-4.00 (m, 4H), 3.79 (s, $3 \mathrm{H}), 3.75-3.82(\mathrm{~m}, 1 \mathrm{H}), 3.62-3.75(\mathrm{~m}, 2 \mathrm{H}), 3.43(\mathrm{~s}, 3 \mathrm{H}), 3.41(\mathrm{~s}, 3 \mathrm{H}), 3.37(\mathrm{~s}, 3 \mathrm{H}), 3.21-3.32(\mathrm{~m}$, 2H), 3.27 (s, 3H), 3.11 (s, 3H), 1.58-2.15 (m, 8H), 1.29 (s, 3H), $1.18(\mathrm{~s}, 3 \mathrm{H}), 1.17$ (s, 3H); ${ }^{13} \mathrm{CNMR}$ $\left(75 \mathrm{MHz}, \mathrm{CDCl}_{3}\right) \delta$ 213.3, 166.7, 159.0, 134.8, 133.0, 130.9, 130.2, 129.6 (2C), 128.3, 118.7, 113.6, 111.0, 96.4, 94.0, 81.7, 80.0, 78.4, 77.8, 77.2, 72.5, 72.0, 71.2, 69.5, 64.9, 64.8, 59.3, 59.1, $57.4,55.5,55.2,51.9,36.5,36.3,35.3,35.0,22.0,19.5,19.0 ;[\alpha]_{\mathrm{D}}{ }^{20}=-26.4\left(\mathrm{c} 0.78, \mathrm{CHCl}_{3}\right)$.

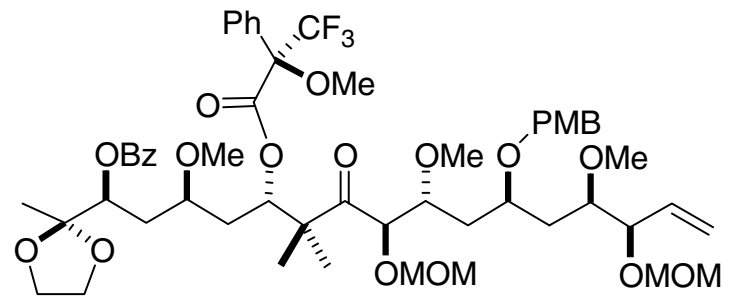

(R)- MTPA ester of 5

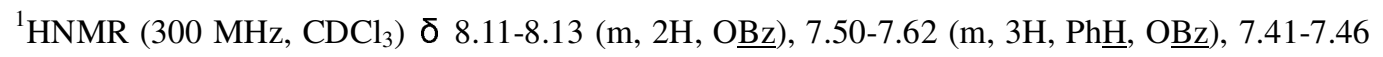
$(\mathrm{m}, 5 \mathrm{H}, \mathrm{Ph} \underline{\mathrm{H}}, \mathrm{OBz}), 7.20\left(\mathrm{~d}, \mathrm{~J}=9.0 \mathrm{~Hz}, 2 \mathrm{H}, \mathrm{OCH}_{2} \mathrm{C}_{6} \underline{\mathrm{H}}_{4} \mathrm{OMe}\right), 6.83(\mathrm{~d}, \mathrm{~J}=9.0 \mathrm{~Hz}, 2 \mathrm{H}$, $\left.\mathrm{OCH}_{2} \mathrm{C}_{6} \underline{\mathrm{H}}_{4} \mathrm{OMe}\right), 5.66-5.82\left(\mathrm{~m}, 2 \mathrm{H}, \mathrm{C} \underline{\mathrm{H}}=\mathrm{CH}_{2}\right.$ and $\left.\mathrm{CH}_{2} \mathrm{C} \underline{\mathrm{H}}(\mathrm{O}-\mathrm{MTPA}) \mathrm{CMe}_{2}\right), 5.26-5.31(\mathrm{~m}, 2 \mathrm{H}$, $\left.\mathrm{CH}=\underline{\mathrm{CH}}_{2}\right), \quad 5.12-5.20 \quad\left(\mathrm{~m}, \quad 1 \mathrm{H}, \quad \mathrm{C} \underline{\mathrm{H}}(\mathrm{OBz}) \mathrm{CH}_{2}\right), \quad 4.70 \quad(\mathrm{~d}, \quad \mathrm{~J}=6.6 \quad \mathrm{~Hz}, \quad \mathrm{~A} \quad$ of $\mathrm{AB}, \quad 1 \mathrm{H}$, $\left.\mathrm{CH}(\mathrm{OMe}) \mathrm{CH}\left(\mathrm{OC}_{2} \mathrm{OCH}_{3}\right) \mathrm{CH}=\mathrm{CH}_{2}\right), 4.67(\mathrm{~d}, \mathrm{~J}=2.1 \mathrm{~Hz}, 1 \mathrm{H}, \mathrm{COC} \underline{\mathrm{H}}(\mathrm{OMOM}) \mathrm{CH}(\mathrm{OMe})), 4.66$ (d, $\mathrm{J}=6.6 \mathrm{~Hz}, \mathrm{~A}^{\prime}$ of $\left.\mathrm{A}^{\prime} \mathrm{B}^{\prime}, 1 \mathrm{H}, \mathrm{COCH}\left(\mathrm{OC}_{2} \mathrm{OMe}\right) \mathrm{CH}(\mathrm{OMe})\right), 4.62\left(\mathrm{~d}, \mathrm{~J}=6.6 \mathrm{~Hz}, \mathrm{~B}^{\prime}\right.$ of $\mathrm{A}^{\prime} \mathrm{B}^{\prime}, 1 \mathrm{H}$, $\left.\mathrm{COCH}\left(\mathrm{OCH}_{2} \mathrm{OMe}\right) \mathrm{CH}(\mathrm{OMe})\right), \quad 4.58 \quad(\mathrm{~d}, \quad \mathrm{~J}=6.6 \quad \mathrm{~Hz}, \quad \mathrm{~B} \quad$ of $\mathrm{AB}, \quad 1 \mathrm{H}$, $\left.\mathrm{CH}(\mathrm{OMe}) \mathrm{CH}\left(\mathrm{OC}_{2} \mathrm{OCH}_{3}\right) \mathrm{CH}=\mathrm{CH}_{2}\right), 4.51\left(\mathrm{~d}, \mathrm{~J}=11.4 \mathrm{~Hz}, \mathrm{~A}\right.$ " of A" $\mathrm{B}$ ", $\left.1 \mathrm{H}, \mathrm{OC}_{2} \mathrm{C}_{6} \mathrm{H}_{4} \mathrm{OMe}\right), 4.25$ (d, J=11.4 Hz, B" of A"B", 1H, OC $\left.\underline{H}_{2} \mathrm{C}_{6} \mathrm{H}_{4} \mathrm{OMe}\right), 4.07-4.11$ (m, 1H, $\left.\underline{\mathrm{H}}(\mathrm{OMOM}) \mathrm{CH}=\mathrm{CH}_{2}\right)$, 3.82-3.96 (m, $\left.4 \mathrm{H}, \quad \mathrm{OCH}_{2} \underline{\mathrm{CH}}_{2} \mathrm{O}\right), \quad 3.78 \quad\left(\mathrm{~s}, \quad 3 \mathrm{H}, \quad \mathrm{OCH}_{2} \mathrm{C}_{6} \mathrm{H}_{4} \mathrm{OMe}\right), \quad 3.60-3.74 \quad(\mathrm{~m}, \quad 2 \mathrm{H}$, $\mathrm{CH}(\mathrm{OMOM}) \mathrm{C} \underline{\mathrm{H}}(\mathrm{OMe}) \mathrm{CH}_{2}$ and $\left.\mathrm{CH}_{2} \mathrm{C} \underline{\mathrm{H}}(\mathrm{OPMB}) \mathrm{CH}_{2}\right), 3.50$ (s, $\left.3 \mathrm{H}, \mathrm{OMe}\right), 3.45$ (s, 3H, OMe), 3.38 (s, 3H, OMe), 3.36 (s, 3H, OMe), 3.33 (s, 3H, $\underline{\mathrm{OMe}}$ ), 3.17-3.30 (m, $2 \mathrm{H}, \mathrm{CH}_{2} \mathrm{C} \underline{\mathrm{H}}(\mathrm{OMe}) \mathrm{CH}_{2}$ and $\left.\mathrm{C} \underline{\mathrm{H}}(\mathrm{OMe}) \mathrm{CH}(\mathrm{OMOM}) \mathrm{CH}=\mathrm{CH}_{2}\right), 3.12(\mathrm{~s}, 3 \mathrm{H}, \mathrm{OMe}), 1.26-1.99\left(\mathrm{~m}, 8 \mathrm{H}, \underline{\mathrm{CH}}_{2} \mathrm{X} 4\right), 1.25(\mathrm{~s}, 3 \mathrm{H}$, $\mathrm{CH}(\mathrm{O}-\mathrm{MTPA}) \underline{\mathrm{Me}}_{2} \mathrm{CO}$ ), 1.24 (s, 3H, , terminal $\left.\underline{\mathrm{Me}}\right), 1.00$ (s, 3H CH$(\mathrm{O}-\mathrm{MTPA}) \underline{\mathrm{Me}}_{2} \mathrm{CO}$ ). 


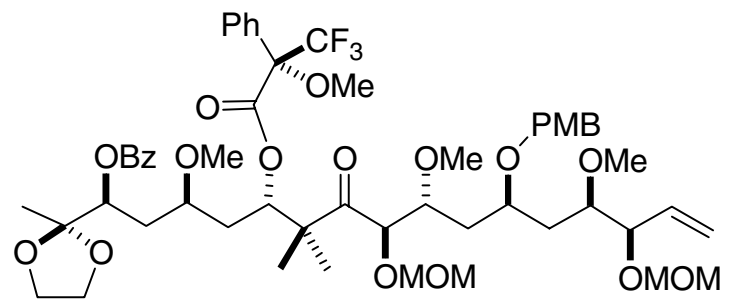

(S)- MTPA ester of 5

${ }^{1} \mathrm{HNMR}\left(300 \mathrm{MHz}, \mathrm{CDCl}_{3}\right) \delta$ 8.11-1.13 (m, 2H, OBz), 7.49-7.58 (m, 3H, $\mathrm{Ph} \underline{\mathrm{H}}$ and $\mathrm{OBz}$ ),

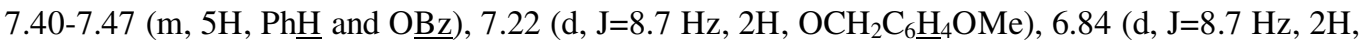
$\left.\mathrm{OCH}_{2} \mathrm{C}_{6} \underline{\mathrm{H}}_{4} \mathrm{OMe}\right), 5.70-5.83$ (m, $2 \mathrm{H}, \mathrm{C} \underline{\mathrm{H}}=\mathrm{CH}_{2}$ and $\left.\mathrm{CH}_{2} \mathrm{C} \underline{\mathrm{H}}(\mathrm{O}-\mathrm{MTPA}) \mathrm{CMe}_{2}\right), 5.27-5.32(\mathrm{~m}, 2 \mathrm{H}$, $\left.\mathrm{CH}=\underline{\mathrm{CH}}_{2}\right), 5.06-5.17\left(\mathrm{~m}, 1 \mathrm{H}, \mathrm{C} \underline{\mathrm{H}}(\mathrm{OBz}) \mathrm{CH}_{2}\right), 4.74(\mathrm{~d}, \mathrm{~J}=2.7 \mathrm{~Hz}, 1 \mathrm{H}, \mathrm{COC} \underline{\mathrm{H}}(\mathrm{OMOM}) \mathrm{CH}(\mathrm{OMe}))$, $4.70\left(\mathrm{~d}, \mathrm{~J}=6.6 \mathrm{~Hz}, \mathrm{~A}\right.$ of $\left.\mathrm{AB}, 1 \mathrm{H}, \mathrm{CH}(\mathrm{OMe}) \mathrm{CH}\left(\mathrm{OC}_{2} \mathrm{OCH}_{3}\right) \mathrm{CH}=\mathrm{CH}_{2}\right), 4.66\left(\mathrm{~d}, \mathrm{~J}=6.9 \mathrm{~Hz}, \mathrm{~A}^{\prime}\right.$ of $\left.\mathrm{A}^{\prime} \mathrm{B}^{\prime}, \quad 1 \mathrm{H}, \quad \mathrm{COCH}\left(\mathrm{OC}_{2} \mathrm{OMe}\right) \mathrm{CH}(\mathrm{OMe})\right), \quad 4.62 \quad\left(\mathrm{~d}, \quad \mathrm{~J}=6.9 \quad \mathrm{~Hz}, \quad \mathrm{~B}^{\prime} \quad\right.$ of $\quad \mathrm{A}^{\prime} \mathrm{B}^{\prime}, \quad 1 \mathrm{H}$, $\left.\mathrm{COCH}\left(\mathrm{OC}_{2} \underline{\mathrm{H}}_{2} \mathrm{OMe}\right) \mathrm{CH}(\mathrm{OMe})\right), \quad 4.58 \quad(\mathrm{~d}, \quad \mathrm{~J}=6.6 \quad \mathrm{~Hz}, \quad \mathrm{~B} \quad$ of $\quad \mathrm{AB}, \quad 1 \mathrm{H}$, $\left.\mathrm{CH}(\mathrm{OMe}) \mathrm{CH}\left(\mathrm{OCH}_{2} \mathrm{OCH}_{3}\right) \mathrm{CH}=\mathrm{CH}_{2}\right), 4.53\left(\mathrm{~d}, \mathrm{~J}=11.4 \mathrm{~Hz}, \mathrm{~A}\right.$ " of A"B", $\left.1 \mathrm{H}, \mathrm{OC}_{2} \mathrm{C}_{6} \mathrm{H}_{4} \mathrm{OMe}\right), 4.26$ (d, J=11.4 Hz, B" of A"B", $\left.1 \mathrm{H}, \mathrm{OC}_{2} \mathrm{C}_{6} \mathrm{H}_{4} \mathrm{OMe}\right), 4.09-4.14$ (m, 1H, $\left.\mathrm{C} \underline{\mathrm{H}}(\mathrm{OMOM}) \mathrm{CH}=\mathrm{CH}_{2}\right)$, 3.80-3.95 (m, 4H, $\left.\underline{\mathrm{OH}}_{2} \underline{\mathrm{CH}}_{2} \mathrm{O}\right), 3.76$ (s, $\left.3 \mathrm{H}, \mathrm{OMe}\right), 3.65-3.73\left(\mathrm{~m}, 2 \mathrm{H}, \mathrm{CH}(\mathrm{OMOM}) \mathrm{C} \underline{\mathrm{H}}(\mathrm{OMe}) \mathrm{CH}_{2}\right.$ and $\mathrm{CH}_{2} \mathrm{C} \underline{\mathrm{H}}(\mathrm{OPMB}) \mathrm{CH}_{2}$ ), 3.53 (s, 3H, $\underline{\mathrm{OMe}}$ ), 3.46 (s, $\left.3 \mathrm{H}, \underline{\mathrm{OMe}}\right), 3.38$ (s, $\left.3 \mathrm{H}, \mathrm{OMe}\right), 3.37$ (s, 3H, $\mathrm{OMe}$ ), 3.33 (s, 3H, OMe), 3.24-3.31 (m, 1H, $\left.\mathrm{CH}_{2} \mathrm{C} \underline{\mathrm{H}}(\mathrm{OMe}) \mathrm{CH}_{2}\right), 3.19$ (s, 3H, $\underline{\mathrm{OMe}}$ ), 3.11-3.22 (m, $\left.1 \mathrm{H}, \quad \mathrm{C} \underline{\mathrm{H}}(\mathrm{OMe}) \mathrm{CH}(\mathrm{OMOM}) \mathrm{CH}=\mathrm{CH}_{2}\right), \quad 1.20-1.98 \quad\left(\mathrm{~m}, \quad 8 \mathrm{H}, \quad \mathrm{C}_{2} \quad \mathrm{X} \quad 4\right), \quad 1.34 \quad(\mathrm{~s}, \quad 3 \mathrm{H}$, $\mathrm{CH}(\mathrm{O}-\mathrm{MTPA}) \mathrm{CMe}_{2} \mathrm{CO}$ ), 1.23 (s, 3H, terminal $\underline{\mathrm{Me}}$ ), 1.06 (s, 3H, $\mathrm{CH}(\mathrm{O}-\mathrm{MTPA}) \mathrm{CMe}{ }_{2} \mathrm{CO}$ ). 


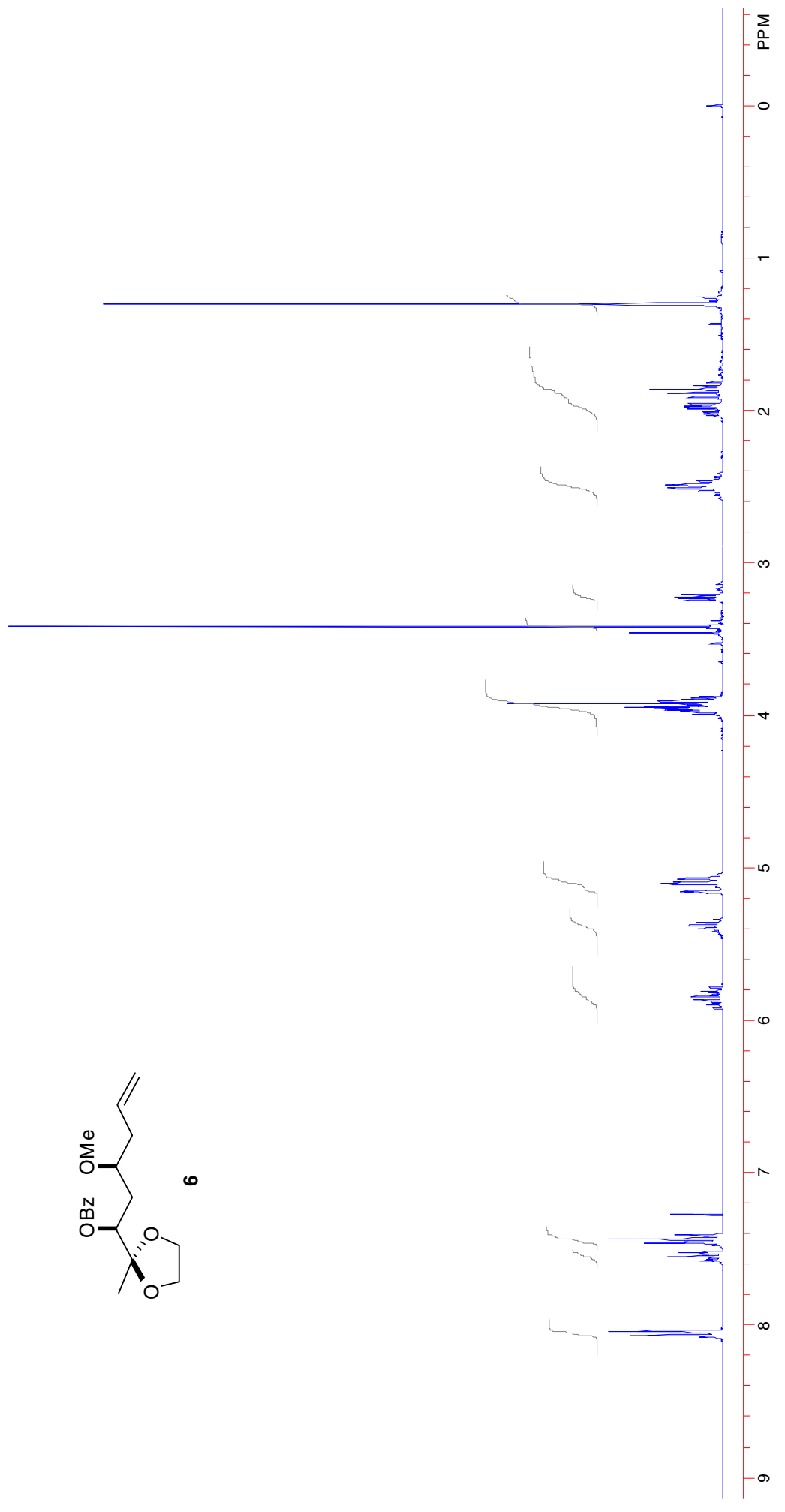




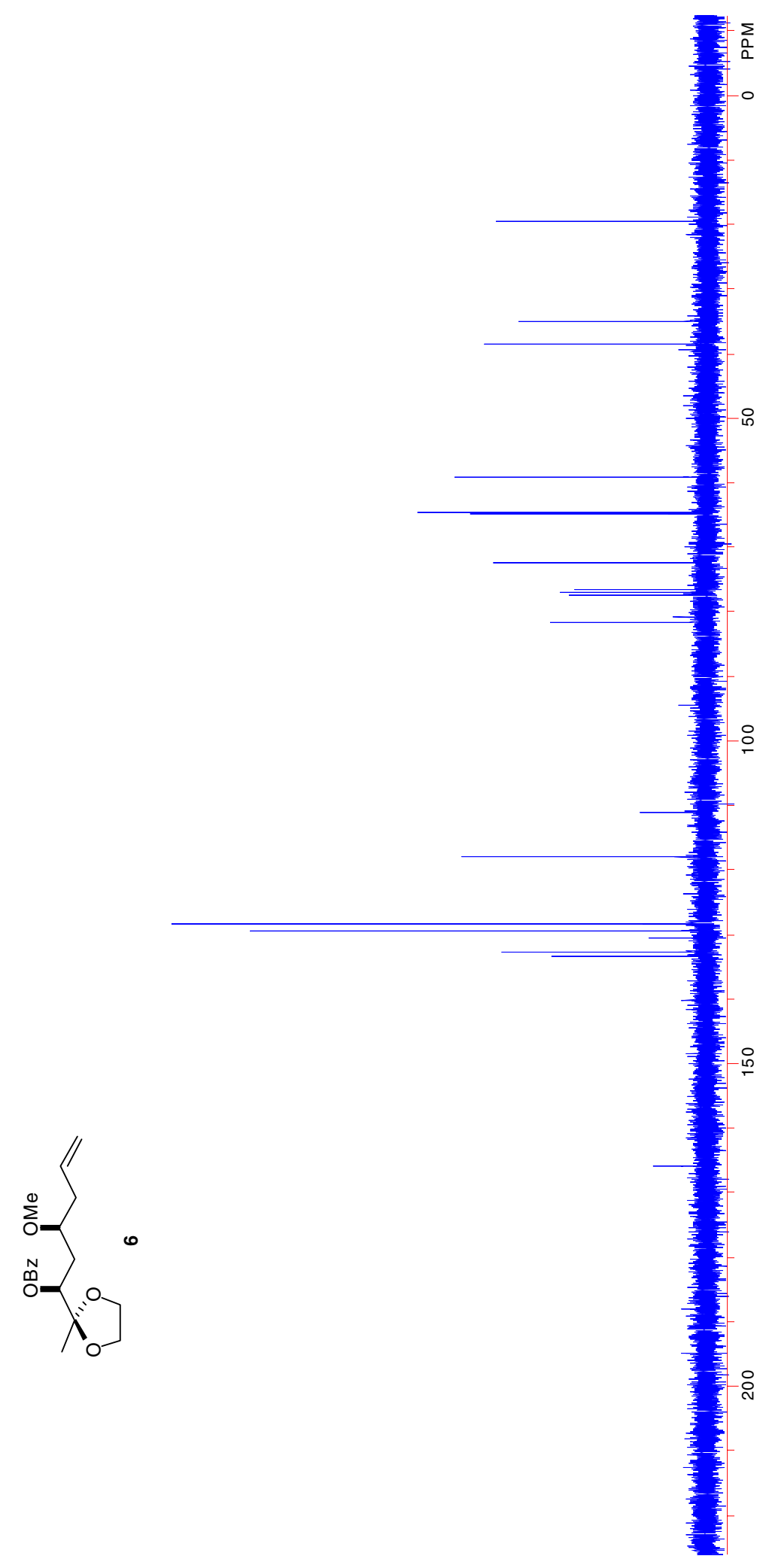




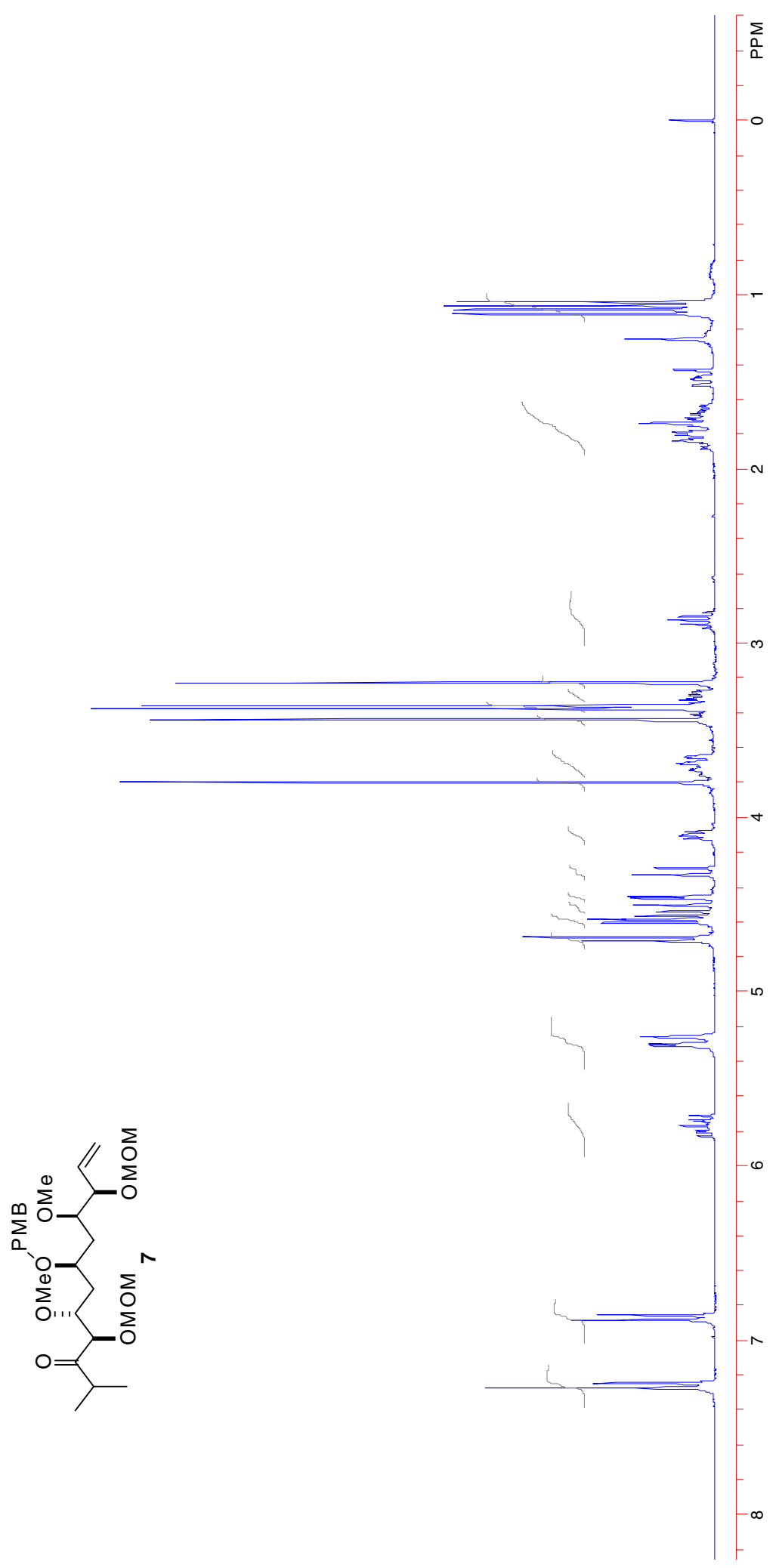



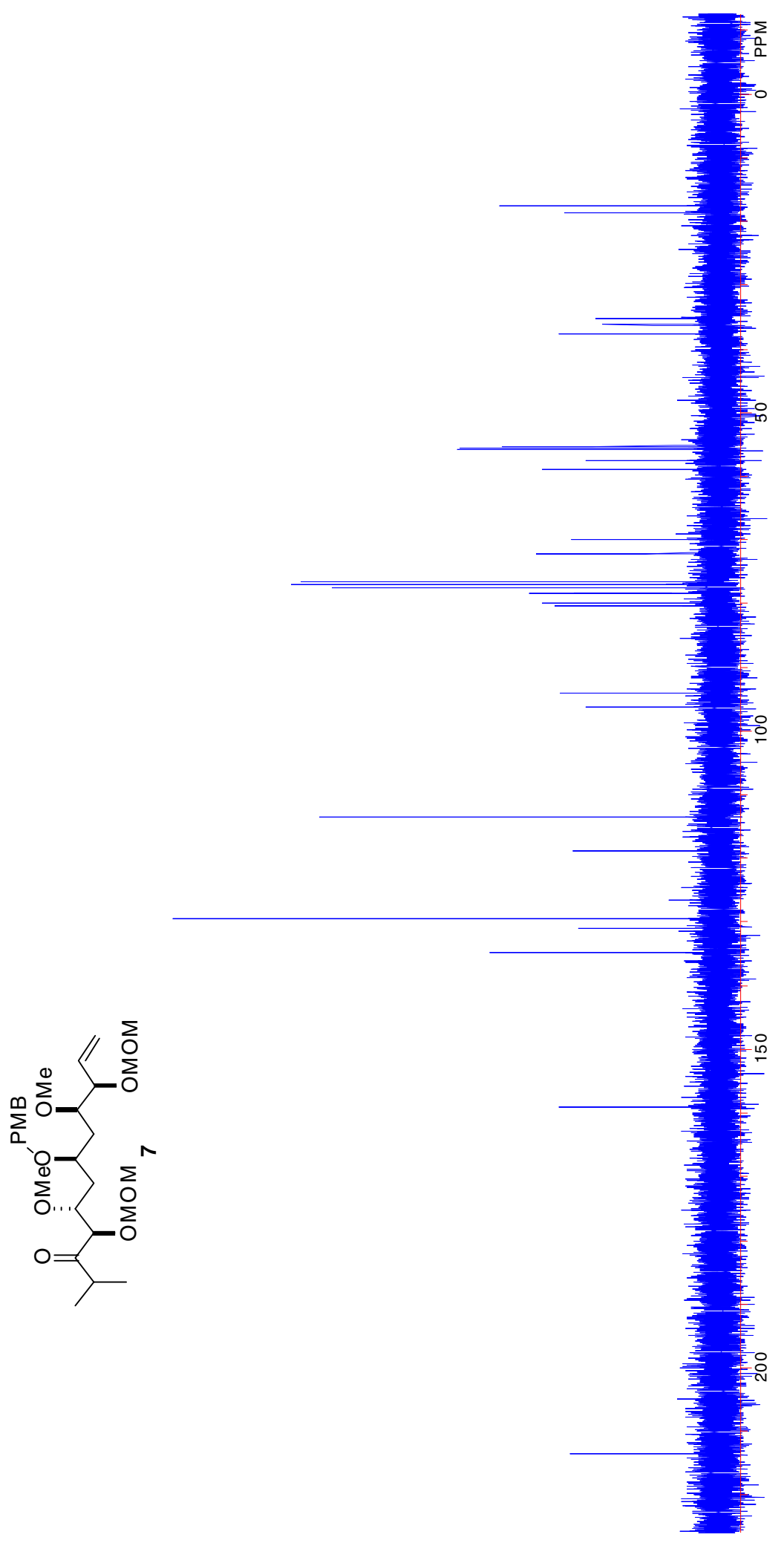


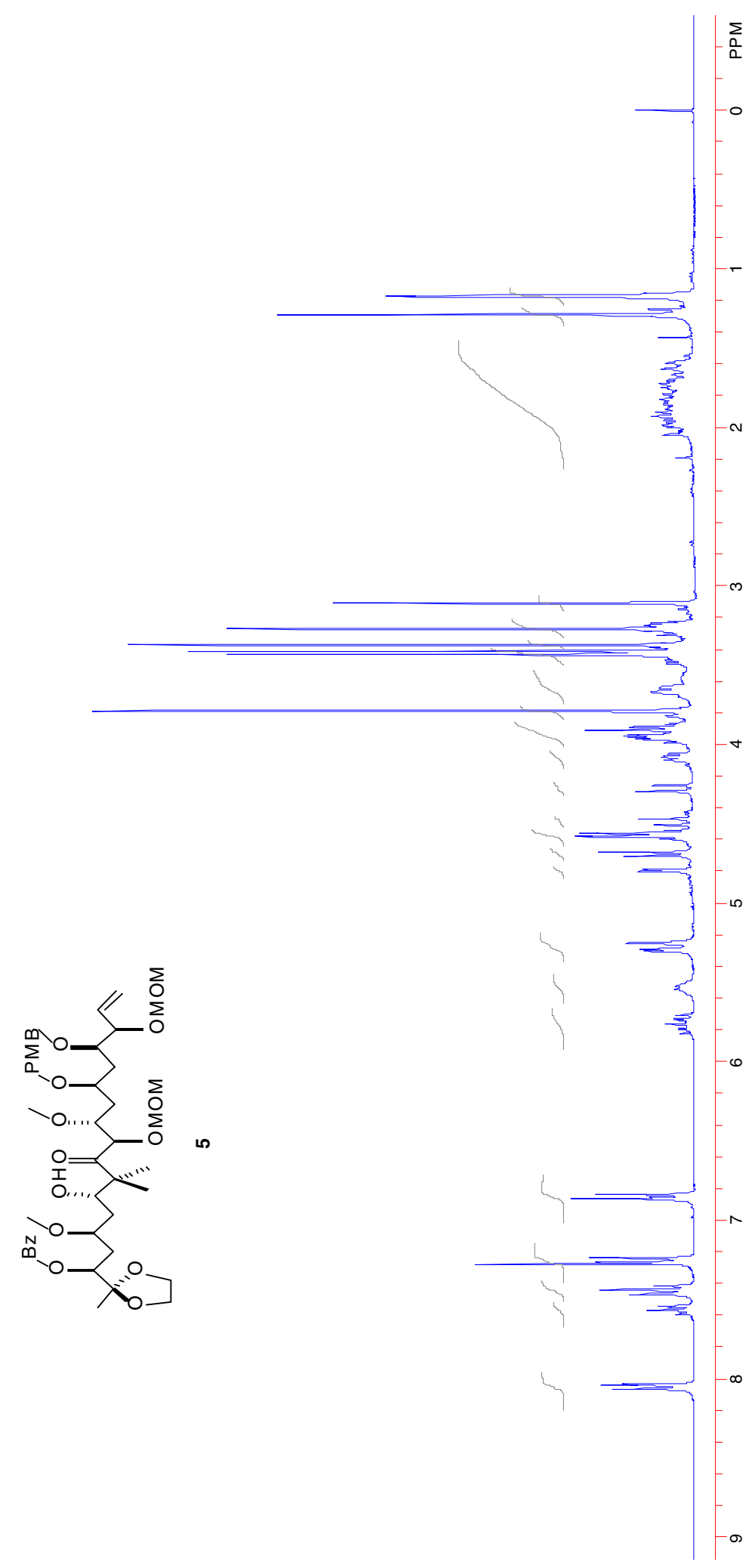




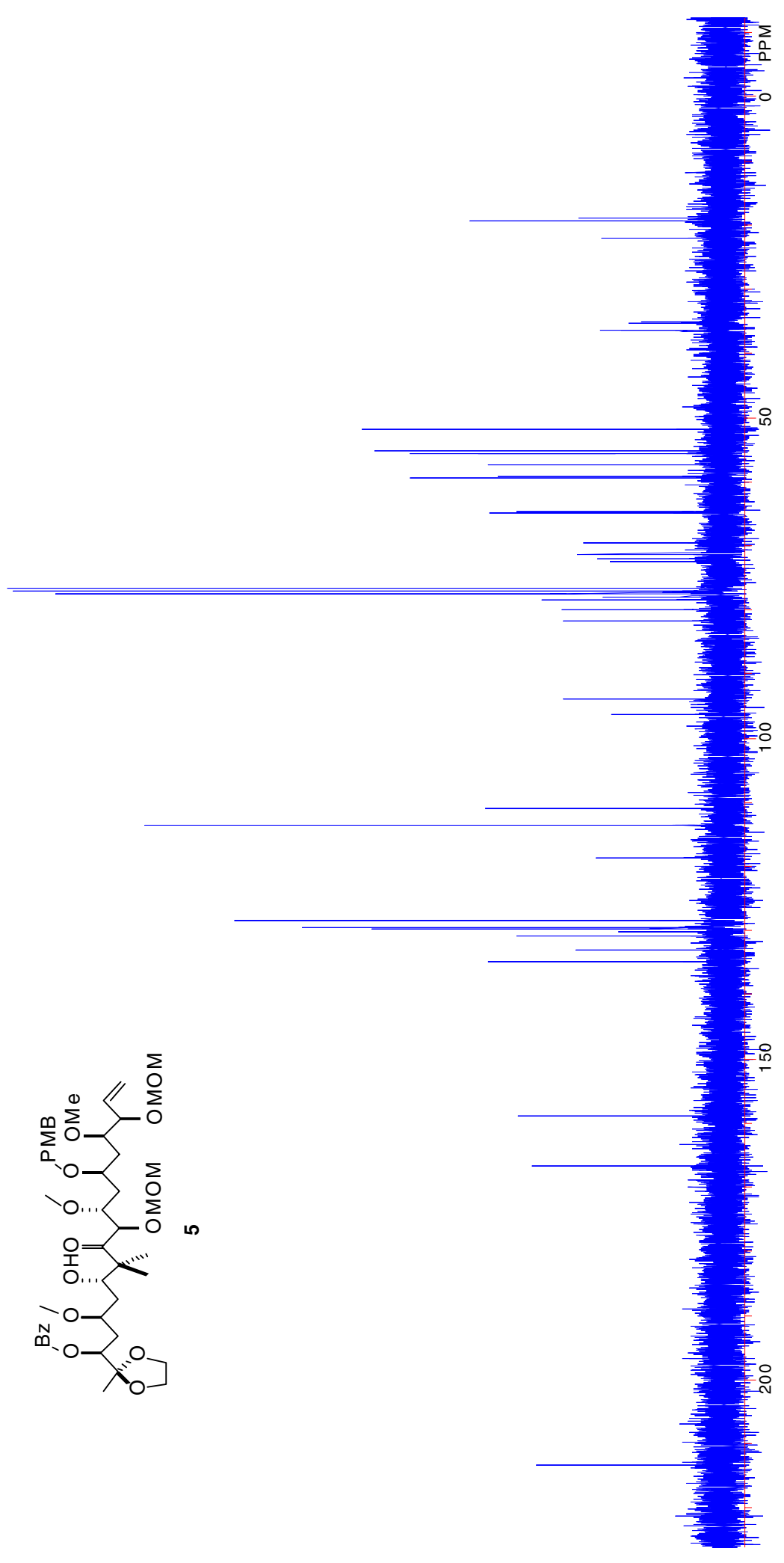

